\title{
OPTICAL PROPERTIES OF GaN EPILAYERS GROWN BY GAS SOURCE MOLECULAR BEAM EPITAXY ON AIN BUFFER LAYER ON (111) Si
}

\author{
M. GODLEWSKI \\ Institute of Physics, Polish Academy of Sciences \\ Al. Lotników 32/46, 02-668 Warsaw, Poland \\ J.P. Bergman, B. Monemar
}

Dept. of Physics and Meas. Technol., Linköping Univ., 58183 Linköping, Sweden

U. Rossner and A. Barski

CEA/Grenoble, Departement de Recherche Fondamentale sur la Matière Condensée/SP2M

17 rue Martyrs, 38054 Grenoble Cedex 9, France

Optical properties of GaN/AlN/Si (111) epilayers grown by MBE are studied. The observed decay transients of excitonic emissions and their temperature dependence is explained by an efficient transfer link between bound and free excitons.

PACS numbers: 71.55.Eq, 71.35.+z, 78.47.+p

\section{Introduction}

A major problem for a wider application of $\mathrm{GaN}$-based structures is the lack of the lattice matched substrates for growing stress free epilayers. Only recently a successful growth of homoepitaxial $\mathrm{GaN} / \mathrm{GaN}$ epilayers was reported $[1,2]$. However, a use of AlN [3] or GaN [4] buffers led to a noticeable improvement of the morphological quality of GaN films grown on lattice mismatched substrates such as sapphire or GaAs and Si.

We report the results of photoluminescence (PL), time-resolved PL and PL kinetics studies of $\mathrm{GaN}$ epilayers grown by gas source molecular beam epitaxy (GSMBE) with AlN buffer layer on (111) Si substrates with $17 \%$ lattice mismatch to a wurtzite phase GaN. A use of Si substrates is motivated by attempt of integrating of Si based electronics with GaN-based light emitting devices. Moreover, Si wafers are cheap, show either $n$ - or $p$-type conductivity and have good morphological quality.

\section{Experimental}

The GaN and AlN films were grown in RIBER MBE 2300 chamber on $p$-type $\left(30 \Omega \mathrm{cm}^{-1}\right.$ ) Si:P (111) substrates. Prior to growth of AlN buffer layers, silicon 
substrates were outgased at $200^{\circ} \mathrm{C}$ and the protective oxide was desorbed at $850^{\circ} \mathrm{C}$. The $2.45 \mathrm{GHz}$ electron cyclotron resonance (ECR) plasma source was used to grow AlN buffer layer at $640^{\circ} \mathrm{C}$. GaN layers were grown by GSMBE with the growth rate about $0.5 \mu \mathrm{m} / \mathrm{h}$ and substrate temperature of $800^{\circ} \mathrm{C}$ by a thermally cracking a high purity ammonia in the presence of gallium. In this respect our approach differs from that of Meng and Perry [5], who have used microwaves in growth of GaN films (ECR-MBE) and reported an increasing concentration of lattice defects with the increasing microwave power.

The PL, PL kinetics and time-resolved PL measurements were performed on experimental setup described elsewhere [6].

\section{Results and discussion}

Figure 1 shows an intensive and broad PL observed at $2 \mathrm{~K}$ at the "edge" part of the emission spectrum. In addition to the "edge" PL, a yellow donor-acceptor pair (DAP) PL was also observed. This PL was relatively weak as compared to the "edge" PL, which is a promising property of our epilayers. Similar "edge" PL with a maximum at about $3.46 \mathrm{eV}$ was observed by Ohtani et al. [7] and Kung et al. [8] and was attributed to the bound excitonic transition (neutral donor bound exciton (DBE)). This PL is asymmetric indicating a contribution of some additional emission(s). Its complex structure could be resolved in the time-resolved PL study (see inset of Fig. 1). Time-resolved PL spectra were measured at $100 \mathrm{ps}$ intervals after the $5 \mathrm{ps}$ long exciting laser pulse. We observed a gradual change of shape of the broad PL band. The additional substructure appears at about $3.414 \mathrm{eV}$, becoming dominant at longer delay times, at $3.456 \mathrm{eV}$, showing a low energy shoulder at about $3.446 \mathrm{eV}$, and at about $3.47-3.49 \mathrm{eV}$, better resolved at longer delay times. This can be observed in the inset of Fig. 1, where the time-resolved PL spectrum measured at $500 \mathrm{ps}$ delay is shown. Based on these

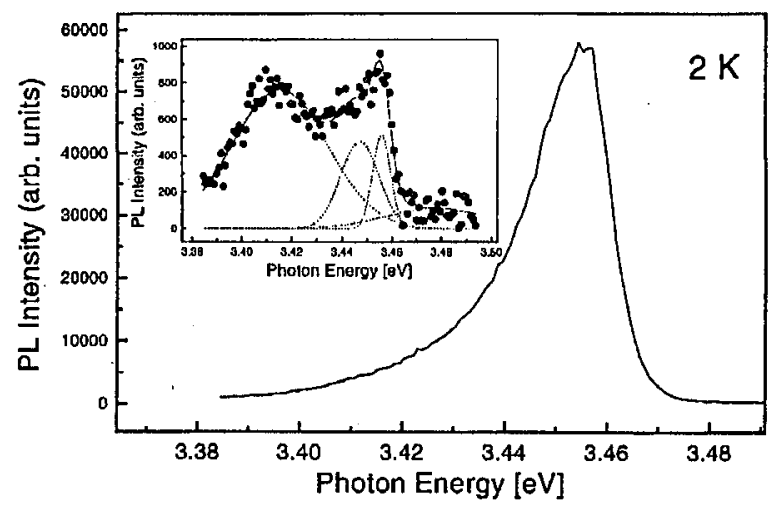

Fig. 1. Photoluminescence spectrum in the "edge" part of the emission of GaN/AlN/Si epilayer measured at $2 \mathrm{~K}$ under nonresonant excitation with a photon energy above GaN band gap. In the inset we show the time-resolved PL spectrum measured at $2 \mathrm{~K}$ with 500 ps delay following 5 ps laser pulse. PL subbands are marked with broken lines. 
results we could decompose the broad PL spectrum into five subbands (a better fit to the experimental results was obtained if we assumed the presence of fifth additional PL decaying fast at longer delay times) with maxima at about $3.47-3.49 \mathrm{eV}$ (free exciton $\mathrm{FE}_{A}$ and/or $\mathrm{FE}_{B}$ ? PL), $3.456 \mathrm{eV}$ (DBE PL), $3.446 \mathrm{eV}$ (neutral acceptor bound exciton (ABE1) PL), $3.436 \mathrm{eV}$ (ABE2 PL) and $3.414 \mathrm{eV}$ (shallow DAP or free-to-bound transition), respectively. The identification of the PL bands, given in the brackets, will be used further in the text. We note here that all the excitonic PL emissions are shifted towards the lower energy by about 16-20 meV compared to the PL energies reported in Refs. [1] and [6] for GaN/GaN epilayer, but the DBE position agrees well with the one reported recently for other homoepitaxial structure [2]. Some shift of the PL position can be related, after Meng and Perry [5], to an unrelaxed strain in our GaN/Si (111) films. It was observed that PL lines in the "edge" part of the emission may shift by as much as $30 \mathrm{meV}$ depending on the stress conditions in the GaN films [9].

PL kinetics measurements were performed for a detection set at different energies within the broad "edge" PL. In Fig. 2 we show a contour plot of the PL intensity versus photon energy and decay time indicating a remarkable property of the PL emission. PL maximum changes its spectral position shifting towards higher (!) energy by about $1 \mathrm{meV}$ during initial $250 \mathrm{ps}$ of the PL decay. The change of the spectral position of the PL was also observed by Smith et al. [10] and was explained by the energy distribution of impurity binding energies, caused by potential fluctuations in $\mathrm{GaN}$ epilayers. However, if such potential fluctuations are present, excitons drift at low temperature towards states of a lower energy [10], and not higher, as is observed by us. The observed shift of the PL emission must thus be of a different origin.

We determined the decay transients of each Gaussian component of the PL from the time-resolved PL study by measuring integrated PL intensities at different delay times. In this way we could avoid limitations of the "conventional" PL decay

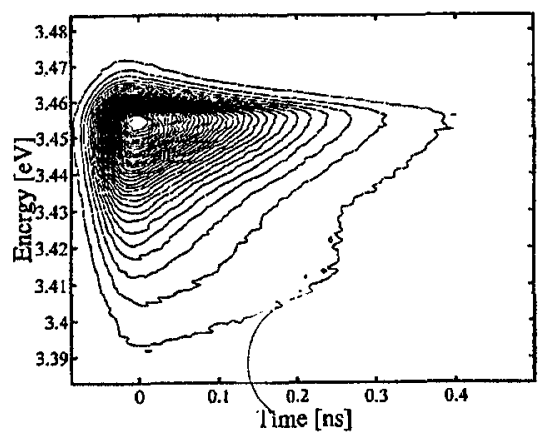

Fig. 2. Two-dimensional contour plot of PL intensity versus photon energy and a decay time of the "edge" part of the PL spectrum measured at $2 \mathrm{~K}$ under nonresonant excitation conditions. The 0 ps time is set at the moment the PL reached its maximum after a 5 ps long laser pulse. 
measurements performed for the overlapping PLs. The FE emission(s), after its initial fast (about $70 \mathrm{ps}$ ) decay, decays then "slowly" with the decay time of about $480 \mathrm{ps}$ at $2 \mathrm{~K}$. This is a decay time much longer than those observed for the DBE (about $115 \mathrm{ps)}$ ), and two ABE PLs: ABE1 (about $110 \mathrm{ps}$ ) and ABE2 (about $80 \mathrm{ps).}$ The observed PL decay times are similar to or longer than the ones reported for GaN/sapphire structures, but are shorter than those measured for excitonic PL emissions for the homoepitaxial GaN epilayer [6].

The temperature dependence of the PL decay was measured. With the increasing temperature PL decay times gradually thermalize. They are about $100 \mathrm{ps}$ for the DBE PL and $80 \mathrm{ps}$ for the ABE1 and ABE2 PL emissions at $40 \mathrm{~K}$. For $50 \mathrm{~K}$ all PL emissions show similar decay time of about 65-70 ps. The "slow" decay component of the FE PL rapidly shortens with increasing temperature and is of about $360 \mathrm{ps}$ for the $20 \mathrm{~K}$ and of about $120 \mathrm{ps}$ for the $40 \mathrm{~K}$.

The following mechanism is proposed to explain the observed time and temperature dependence of the PL emission. We assume that bound excitons can convert into free excitons by tunneling at low temperature, or by phonon-assisted tunneling and thermal dissociation at increased temperatures. Assuming that transfer link between bound and free excitons is efficient, we estimate exciton tunneling rate to be (at $2 \mathrm{~K}$ ) about $10^{10} \mathrm{~s}^{-1}$ for the ABE-DBE transfer and about $2 \times 10^{9} \mathrm{~s}^{-1}$ for the DBE-FE transfer. These values are the upper limits for the proposed transfer mechanism since other nonradiative recombination processes cannot be excluded. The mechanism explains why at increased temperatures (above 40 $\mathrm{K})$ the decay times of bound excitons become equal to the decay time of free excitons and also explains the observed drift of the PL maximum towards the higher energy during the decay time.

\section{References}

[1] K. Pakula, A. Wysmolek, K.P. Korona, J.M. Baranowski, R. Stepniewski, I. Grzegory, M. Boćkowski, J. Jun, S. Krukowski, M. Wróblewski, S. Porowski, Solid State Commun. 97, 919 (1996).

[2] F.A. Ponce, D.P. Bour, W. Gotz, N.M. Johnson, H.I. Helava, I. Grzegory, J. Jun, S. Porowski, Appl. Phys. Lett. 68, 917 (1996).

[3] H. Amano, T. Akasaki, K. Hiramatsu, N. Koide, N. Sawaki, Thin Solid Films 163, 415 (1988).

[4] S. Nakamura, Jpn. J. Appl. Phys. Lett. 30, L1705 (1991).

[5] W.J. Meng, T.A. Perry, J. Appl. Phys. 76, 7824 (1994).

[6] M. Godlewski, A. Wysmołek, K. Pakuła, J.M. Baranowski, I. Grzegory, J. Jun, S. Porowski, J.P. Bergman, B. Monemar, in: Proc. Int. Symp. on Blue Laser and Light Emitting Diodes, Chiba (Japan) 1996, Eds. A. Yoshikawa, K. Kishino, M. Kobayashi, T. Yasuda, Omsha, Ltd., Tokyo, 1996, p.356.

[7] A. Ohtani, K.S. Stevens, R. Beresford, Appl. Phys. Lett. 65, 61 (1994).

[8] P. Kung, A. Sexler, X. Zhang, D. Walker, T.C. Wang, I. Ferguson, M. Razeghi, Appl. Phys. Lett. 66, 2958 (1995).

[9] H. Amano, K. Hiramatsu, I. Akasaki, Jpn. J. Appl. Phys. 27, L1384 (1988).

[10] M. Sinith, G.D. Chen, C.J. Sun, Appl. Phys. Lett. 67, 3295 (1995). 